\title{
Intracranial Acute Subdural Hematoma: Is there a Space for a Conservative Treatment in the Elderly?
}

\author{
Tacconi Leonello* and Spinelli Roberto \\ Department of Neurosurgery, Azienda Universitaria Integrata, Italy
}

Submission: February 09, 2018; Published: March 09, 2018

*Corresponding author: Tacconi Leonello MD, FRCS Ed (SN), Department of Neurosurgery, Azienda Universitaria Integrata, Strada di Fiume 447, 34100, Trieste, Italy, Tel: +390403994466; Email: leonello.tacconi@asuits.sanita.fvg.it

Keywords: Acute; Subdural; Elderly; Age; Conservative

\section{Introduction}

Acute subdural hematoma (ASH) is a collection of acute blood into the subarachnoid space. The most common cause of ASH is head trauma with consequent rupture of a cortical blood vessel, commonly a bridging vein. Their treatment is a surgical emergency and the outcome is related to the age, size of hematoma as well as to the rapidity of the surgical treatment. Guidelines have already clarified the necessity of a surgical approach in specific cases of ASH. If the decision of what to do can be relatively easy in young and middle age patients it can become problematic in elderly patients who have also a greater number of comorbidities. With the aim to see if there are some patients with ASH which can be treated conservatively despite the guidelines indications we have collected retrospectively 17 cases with ASH who we have tried to treat conservatively. We will discuss the outcome and complication along with our philosophy in these specific cases.

\section{Material and Methods}

Our Department provides the service for a geographical region with high prevalence of elderly people. For this reason we were able, among a population of 88 operated ASH admitted to our unit in a 4 years period (2011-2015) to selected 21 cases treated conservatively. Among these we have chosen 17 patients whose age was 80 and older. The mean age of our series was 84 years old (range 80-89). Male to female ratio was 1.1:1. Right side ASHs were slightly more frequent (55\%). In 15 cases (88\%), ASH developed in the whole convexity (fronto-temporo-parietal); moreover, there was 1 occipital and 1 interhemispheric ASH. All ASHs had a thickness greater than $10 \mathrm{~mm}$, with a mean of $12 \mathrm{~mm}$ (range 11-15). The mean midline shift was $4 \mathrm{~mm}$ (range 3-10). Upon clinical examination, on admission to our Department the mean GCS score was 12 (range 10-13). All Patients were in treatment with antiplatelet or anticoagulant: 8 with warfarin, 3 with acenocoumarol, 2 with new oral anticoagulants (NOACs) and 4 with antiplatelet.

All 17 patients were initially treated conservatively. Three underwent an emergency craniotomy and evacuation of the ASH a few days after admission (range 24-56 hours), (Figure $1 \mathrm{~A} \& 1 \mathrm{~B}$ ) because of neurological deterioration and one patient died two days after admission for progressive increasing of the intracranial pressure. Among the rest of the patients treated medically (Figure 2) (13 patients) all out of two (11 patients) were transferred to a rehabilitation center or nursing home after a median of hospital staying of 23 days (range between 21 and 33 days). The other two were operated on for a chronic subdural hematoma (CSH) and a week postoperatively they were transferred back to the referring hospital. Also the three patients operated on for the ASH were transferred to a rehabilitation unit, just after a week from surgery. Among the 11 patients treated conservatively (Figure 2) and discharged from the neurosurgical ward, five (45\%) were readmitted for the treatment of a developed CSH. All patients received IV steroids for a mean of 4 days (range 3-7) at the admission. In order to prevent thromboembolic events due to poor mobilization, we choose to start low molecular weight heparin (LMWH) 2 days after admission, but only in case the CT images were stable. All patients underwent a 12 hours post admission follow up CT scan or earlier in case of deterioration. A second scan was 
performed after 48-72 hours and then after a week and before the discharge as well as after 2, 4 and 6 weeks in order to detect the transformation from acute to $\mathrm{CSH}$ and any evidence of mass effect.

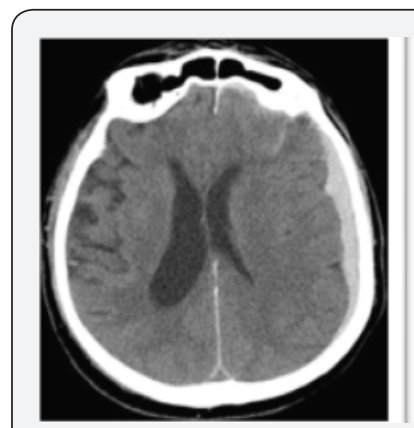

A

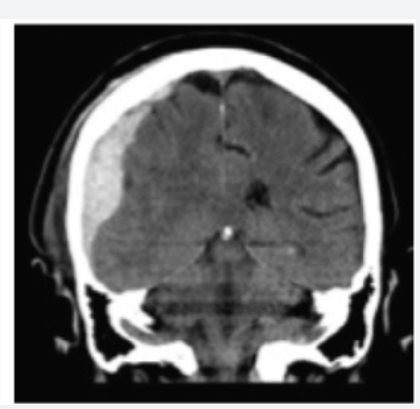

B
Figure 1: (1A: Axial view) and (1B: coronal view) Brain CT scan showing a right ASH of the convexity. Due to neurological deterioration occurred 48 hours after admission, the patient underwent a surgical evacuation of the hematoma.

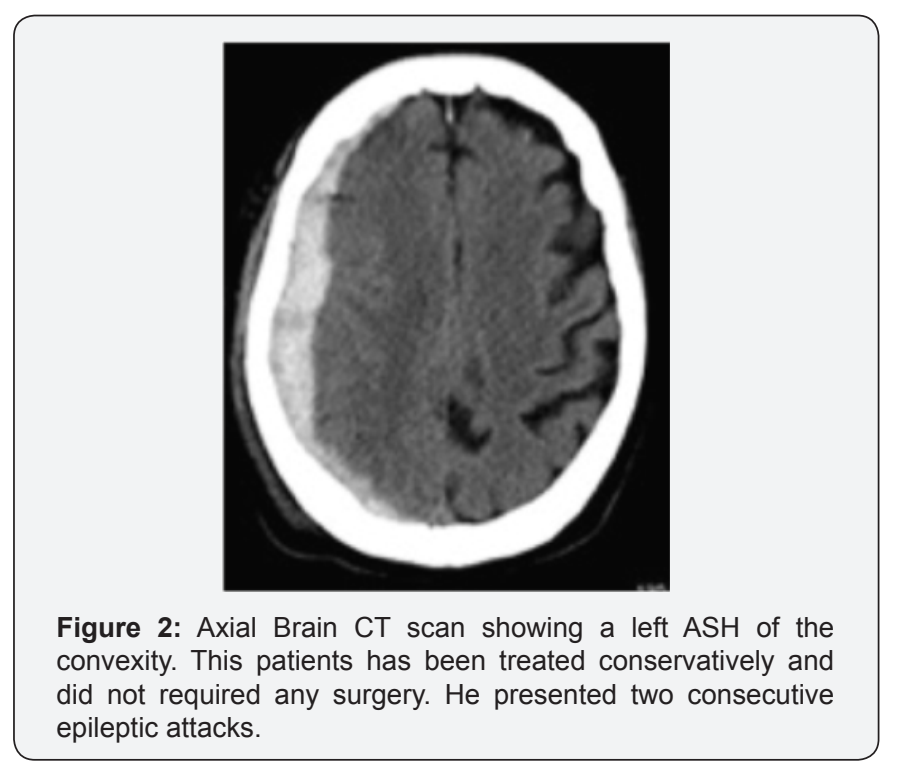

\section{Results}

Among the 17 patients we tried to treat conservatively 1 died, 3 underwent an emergency craniotomy and evacuation of ASH. Thirteen were continuously treated conservatively. Among these, two patients were operated on for a CSH during the same in-hospital staying. One after 28 days and the other after 33 days from the trauma. Among the 11 patients discharged from the neurosurgical care, 5 were operated on for a CSH during a second admission and discharged back after a median time of 5 days; the remained 6 cases out of 17 (35,2\%) were treated conservatively and at a median follow up of 11 months (range 8-14 months) they have a GOS between 2 and 5 (mean 3). At the same follow up the mean GOS for surgically treated patients was 2,9 (range 2-4).

Two out of three patients (66\%) treated in the acute phase developed a urinary infection during hospitalization. Among patient treated conservatively (11 cases), seven developed urinary infection, other two pulmonary infection, one patients with an history of chronic atrial fibrillation went on to acute cardiac failure and 3 suffered from epileptic seizures. All these complications were treated successfully with antibiotics and medical treatment. Surgically treated patient underwent a median of 4 follow up CT scan (range 3-4) while conservative treated patients underwent a higher number of CT scans: a median of 6 (range 5-8). The overall mean hospitalization time was 24 days (range 14-38). The mean length of hospitalization after the surgical treatment was 10 days (range 7-12) while for conservative treated patients it was 26 days (range 24-33).

\section{Discussion}

Among young persons, traumatic brain injury (TBI) is still the leading cause of morbidity and mortality. Worldwide, about 10 million of people per year experience a head injury that lead to hospitalization, residual deficits or death. About $70 \%$ of hospitalizations for ASH in the USA are for over 60 years old patients, and only about $40 \%$ of over 65 years old patients that underwent surgical treatment for ASH have a good outcome $[1,2]$. The overall mortality after a brain trauma reaches the $9 \%$ of cases. Incidence of TBI is higher in children and elderly people and the main causes are motor vehicle accident, falls and gunshot wounds $[3,4]$. Improving of health-care has determined an important rising in the patient's age and consequently an increased incidence of ASHs in elderly. In this population, brain atrophy increases the subdural space and a teared bridging vein may be more prone to bleed after also a mild trauma. Furthermore the chronic use of anticoagulant and antiplatelet therapies increases the risk of acute bleeding. According to some previous studies, common indications for surgical evacuation of an ASH were a midline shift more than $5 \mathrm{~mm}$ with a GCS score less than 15 and a thickness greater than $10 \mathrm{~mm}[5,6]$.

If we look to more recent Guidelines, any ASH with a thickness greater than $10 \mathrm{~mm}$ or a midline shift more than $5 \mathrm{~mm}$ should undergo surgical evacuation regardless the GCS score [7]. Surgery should also be offered with a thickness less than $10 \mathrm{~mm}$ and a midline shift less than $5 \mathrm{~mm}$, in case of GCS less than 9 which tends to decrease $>2$ points and/or when ICP is greater than $20 \mathrm{~mm} \mathrm{Hg}$ and/or when there are signs of cerebral herniation (ex: pupillary abnormalities). If guidelines are generally very useful they may not help in the decision making in very old patients. In these specific age group, due to agerelated preoperative co morbidities and a higher risk of periand postoperative medical complications, neurosurgeons should therefore consider not only the hematoma thickness, the midline shift and the GCS score in order to choose for the most tailored management (conservative vs surgical) but also the amount of brain atrophy, the previous clinical status of the patient, any anesthesiology risks as well as his/her will. Sometimes, even though according to guidelines the features of the hematoma would require a surgical evacuation, a conservative approach 


\section{Open Access Journal of Neurology \& Neurosurgery}

and a closed clinical monitoring may be used, offering surgery only in case of delayed neurological deterioration [8-10].

Indeed, due to increasing of subdural spaces related to brain atrophy, it is common evidence that an old patient with a huge ASH may be neurologically intact or have only minor deficits. In his report regarding the outcome after head trauma in an elderly population in developing countries (more than 65 years old), Prasad described a rate of hospitalization for non fatal head injury of about 155 cases per 100000 population per year [11]. The author identified diabetes mellitus, hypertension, ischemic heart disease and chronic renal disease as the most frequent comorbidity. Brain contusion ( $40 \%$ of cases) and ASD (25\% of cases) were the most common finding on brain imaging. Due to tight adhesion of the dura to the skull, acute epidural hematomas are rare in elderly people. Age has been identified as an independent predictor of poor outcome and the mortality rate also after mild head trauma in elderly patients is quite high (around 55\%, according to different studies) and a poor outcome (GOS 1-3) reaches a $27 \%$ of cases $(45 \%$ considering only patients older than 75 years old). McGinity described the outcome after evacuation of ASD in patients older than 80 years old [12]. The mean day spent in the ICU was about 10 days, with a mean hospitalization of 13.2 days. Interestingly, the author noted that, although all patients with a preoperative treatment with Clopidogrel had a poor outcome, the use of ant platelet or anticoagulant wasn't related with the clinical results. The most common post operative complications were pneumonias, DVT, pulmonary embolisms, seizures, cardiac arrhythmia, and urinary tract infection. The median number of complication per patient ranged between 2.5 to 3 during the hospitalization. A good outcome (GOS 4-5) was achieved in only $18 \%$ of cases. Although a good outcome was related with a lower mean hematoma thickness ( $17 \mathrm{vs} 20 \mathrm{~mm}$ ), lower mean midline shift ( $10 \mathrm{vs} 14 \mathrm{~mm}$ ) and lower mean GCS at admission (14.5 vs 10), the difference in thickness between patients with a good and poor outcome did not reach a statistic significance and the difference of the midline shift and the GCS at admission reach only a trend to significance. Moreover, patients surgically treated after an initial conservative management, had a better outcome than patients operated emergently, although this data didn't reach a statistical significance. Bajsarowicz, in his large series investigated the risk factors correlates for the necessity of a delayed surgical evacuation in patients with an ASH managed conservatively at the admission [13]. In this study, only $6.5 \%$ of patients required a delayed surgical intervention. The major reason determining the surgical approach (after a median of 9.5 days) was an increase of the ASH (73.8\%); other minor causes were an increase in ICP $(23.8 \%)$ and status epilepticus (2.4\%). The authors identified as risk factors for a delayed surgery the ASH size, the midline shift, the hematoma location at the convexity, and positive history for repetitive falls and alcohol abuse. A good outcome was achieved in $77 \%$ of patients. Interestingly, although cerebral atrophy is an already well described risk factor for the development of a CSH, Bajsarowicz did not identified atrophy as a risk factor for the progression of the ASHs. Similarly, in his retrospective study, Kim analyzed the risk factors associated with a delayed surgical evacuation of ASHs after a mild head injury treated conservatively on admission. [13] Thirty-five per cent of patients had undergone a deferred surgery, after a mean of 17 days. The volume of the hematoma and the midline shift were identified as negative prognostic factors. Even though ASH occurs more frequently in elderly, neither increased age nor brain atrophy were identified as risk factors for delayed enlargement of the hematoma and necessity of surgical evacuation.

A proposed theory is related to the increasing subarachnoid compartment secondary with the brain atrophy: tears of the subarachnoid membrane after the head trauma may play a role in the gradual resolution of the hematoma by diluting it, reducing its mass effect and eventually lowering the risk of the hematoma progression [13-15]. In the same study, Kim reported a reduced incidence of ASDH progression in case of concurrent brain contusions and SAH. According to the author, brain contusions with perilesional edema can increase brain swelling which can act as a counter pressure that prevent subdural hematoma enlargement by reducing the subdural space [16]. McGinity found a good outcome after an early evacuation of an ASH in patients older than 80 years old in only $18 \%$ of cases [17]. The author managed conservatively 13 patients with an admission GCS score of $14-15$ and all but 1 patient suffered from a neurological deterioration requiring a delayed surgical intervention. Unfortunately thickness and midline shift of these cases were not described but, although without statistical significance, these patients had better outcome than patients that had undergone an emergent surgical intervention on admission. One patient in our series died very soon after admission. It was an 89 year old man, admitted with a GCS of 12 (E3V4M5). The patient had a medical history of relevant cardiac and pulmonary comorbidities and an ongoing pulmonary neoplasia. In this patient, despite progressive neurological deterioration due to growing of ASH thickness and increasing midline shift, we choose not to perform surgery and the patient passed away 2 days later after admission.

Among the 16 patients treated conservatively, 7 underwent delayed uneventful double burr hole for evacuation of $\mathrm{CSH}$ (two during the time they were still inpatients in our ward). We did not find any difference, in terms of GOS between those three patients operated on in the acute phase and the seven operated on for a CSH in a delay fashion. Also the length of inpatient staying between surgically and not surgically treated patients was very similar. If we consider, instead, the length of stay in a specialized ward (neurosurgical or high depended unit) we found a significant difference between those who had been operated and those treated conservatively: respectively 7 days versus 23 days (this could rise the question of economical hospital issues). Overall, conservative group experienced 13 


\section{Open Access Journal of Neurology \& Neurosurgery}

complications in 11 patients ( 7 urinary infections, 2 pulmonary infections and 1 acute cardiac failure and 3 seizures), whereas surgical group experienced 2 urinary infections in two out of three patients.

Although our study has a major limitations because of the small numbers of patient and its retrospective nature we would like to make some considerations:

A. The outcome either with or without surgery is very poor in this age group;

B. The number of CT scans which is normally performed in patient with a surgical intervention is less than in patient treated conservatively;

C. The median in-patient staying is very similar in both groups but if we consider specifically the in hospital staying of the operated patients in a high specialized environment (neurosurgical wards or high depended unit) this is much less when compared with the patient in-staying of the group treated without surgery.

D. The medical complications seems to be higher in the conservative treated group;

E. An operation for a secondary developed CSH is less traumatic and faster for an old patients and can be performed also under local anesthetic.

As far as we know there is not in the literature a prospective randomized study which compares the outcome of conservative and surgically treated of ASH in elderly patients. Therefore, without specific guidelines focused on this age group, it is our opinion that the decision of what to do in ASH in elderly must be chosen facing all the aspect and variables of the patient: age, comorbidities, GCS, possible ongoing deterioration during hospitalization, previous medical history, radiological features of the ASH and last but very important point the patient's wills. Probably a conservative management whenever possible should be attempted. Concurrently we must start an active and careful medical and nursing monitoring in order to prevent the onset of any complications.

\section{Conclusion}

Our philosophy is therefore to delay any surgery as long as we can, waiting for a possible chronic transformation of the hematoma that could be successfully treated at a later stage with a less invasive surgical procedure even in local anesthesia $[17,18]$. In the elderly patients a good outcome is related not only to the management of the ASH but also to the prevention and a prompt treatment of any medical complications during the hospitalization.

\section{References}

1. McGinity MJ, Michalek JE, Rodriguez JS, Floyd JR (2017) Surgical evacuation of acute subdural hematoma in octogenarians: a ten-year experience from a single trauma center. Br J Neurosurg 31: 714-717.

2. Taussky P, Hidalgo ET, Landolt H, Fandino J (2012) Age and salvageability: Analysis of outcome of patients older than 65 years undergoing craniotomy for acute traumatic subdural hematoma. World Neurosurgery 78(3-4): 306-311.

3. Hatashita S, Koga N, Hosaka Y, Takagi S (1993) Acute subdural hematoma: severity of injury, surgical intervention, and mortality. Neurol Med Chir (Tokyo) 33: 13-18.

4. Howard MA, Gross AS, Dacey RG, Winn HR (1989) Acute subdural hematomas: an age-dependent clinical entity. JNS 71(6): 858-863

5. Mathew P, Olunya-Oluoch DL, Condon BR, Bullock R (1993) Acute subdural haematoma in the conscious patient: Outcome with inital non-operative management. Acta Neurochir (Wien) 121: 100-108.

6. Wong CW (1995) Criteria for conservative treatment of supratentorial acute subdural haematomas. Acta Neurochir (Wien)135: 38-43.

7. Bullock MR, Chesnut R, Ghajar J, Gordon D, Hartl R, et al. (2006) Surgical management of acute subdural hematomas. Neurosurgery 58(3 Suppl): S16-S24

8. Cagetti B, Cossu M, Pau A, Rivano C, Viale G (1992) The outcome from acute subdural and epidural intracranial haematomas in very elderly patients. Br J Neurosur 6: 227-231.

9. Kwon H, Choi KS, Yi HJ, Chun HJ, Lee YJ, et al. (2017) Risk factors of delayed surgical intervention after conservatively treated acute traumatic subdural hematoma. J Korean Neurosurg Soc 60(6): 723729.

10. Towers WS, Kurtom KH (2014) Spontaneous resolution of large acute subdural hematoma and the value of neurological exam in conservative management of high risk patients. Clin Neurol Neurosurg 118: 98-100.

11. Prasad GL, Anmol N, Menon GR (2017) Outcome of traumatic brain injury in elderly population: A tertiary centre experience from a developing country. World Neurosurg 111: e228-e234.

12. Paul B, Ipshita P, Julie L, Rajeet Singh S, Mitra F, et al. (2015) Nonsurgical acute traumatic subdural hematoma: what is the risk? JNS 123: 11761183.

13. Gan Q, Zhao H, Zhang H, You C (2017) Rapid spontaneously resolving acute subdural hematoma. J of Craniofac Surg 28: 287-289.

14. Takeshi M, Takahide S, Yoshinari O, Sakaki T (1997) Rapid resolution of symptomatic acute subdural hematoma: case report. Surg Neurol 48(2): 193-196.

15. Wong ST, Yuen MK, Fok KF, Yuen SC, Yam KY, et al. (2009) Redistribution of hematoma to spinal subdural space as a mechanism for the rapid spontaneous resolution of posttraumatic intracranial acute subdural hematoma: case report. Surg Neurol 71(1): 99-102.

16. Kim BJ, Park KJ, Park DH, Lim DJ, Kwon TH, et al. (2014) Risk factors of delayed surgical evacuation for initially nonoperative acute subdural hematomas following mild head injury. Acta Neurochir (Wien) 156(8): 1605-1613.

17. Hanif S, Abodunde O, Ali Z, Pidgeon C (2009) Age related outcome in acute subdural haematoma following traumatic head injury. Ir Med J 102: 255-257.

18. Lee JJ, Won Y, Yang T, Kim S, Choi C, et al. (2015) Risk Factors of Chronic Subdural Hematoma Progression after Conservative Management of Cases with Initially Acute Subdural Hematoma. Korean J Neurotrauma 11(2): 52-57. 
Your next submission with Juniper Publishers will reach you the below assets

- Quality Editorial service

- Swift Peer Review

- Reprints availability

- E-prints Service

- Manuscript Podcast for convenient understanding

- Global attainment for your research

- Manuscript accessibility in different formats

( Pdf, E-pub, Full Text, Audio)

- Unceasing customer service

Track the below URL for one-step submission https://juniperpublishers.com/online-submission.php 